\title{
Adaptive Fuzzy Gain of Power System Stabilizer to Improve the Global Stability
}

\author{
Mohammed Mekhanet ${ }^{\star 1}$, Lakhdar Mokrani ${ }^{2}$, Aissa Ameur ${ }^{3}$, Yacine Attia ${ }^{4}$ \\ 1,2,3 Electrical Engineering Department, Amar Telidji University of Laghouat, B.P 37G-Laghouat, Algeria \\ ${ }^{4}$ Electrical Engineering Department, University of Medea, CitéAin D'hab, 26000, Médéa, Algeria \\ *Corresponding author, e-mail: mohamed.mekhanet@yahoo.fr
}

\begin{abstract}
The lead-lag power system stabilizer has several parameters to be optimized.In fact, the number of these latter increases with the number of generators constituting the multi-machine system. In this work, we propose anew approach of an adaptive and robust PSS; it achieves encouraging results by adjusting the gain using fuzzy logic and in the same time we use the same PSSs for each machine. In the first place, we could check that the gain is among the most critical parameters of the lead lag PSS. The parameters are globally optimized by the genetic algorithm, after that an expertise on the speed and the gain variations allow the value prediction according to the velocity deviation. To validate our results, a robustness test was made on a multimachine system IEEE (3 machines 9 bus), for different loads and the results showed good performance and robustness of the presented PSS.
\end{abstract}

Keywords: fuzzy logic, adaptive gain, genetic algorithm, auto adaptation, simulink/matlab

\section{Introduction}

Low frequency oscillations are the operational constraints that limit the transmission of electrical energy. In such a scenario, the power control system play a significant role, it may contribute either generating a positive or negative damping. The automatic voltage regulators (AVR) can be the source of generating negative amortization [1]. High gain of the voltage regulator can lead to poor damp or generation of negative oscillations [2]. This problem has led to the addition of a signal in the excitation control loop generated by a device called a Power System Stabilizer (PSS) to dampen the negative oscillations. The essential problem is to provide an adaptive PSS for every operating point. For the determination of the PSS parameters, several methods have been treated by literature, including the adaptive methods using artificial intelligence such as neural networks [3-4], or neuro-fuzzy [5-7]. The Fuzzy logic controller $(F L C)$ is seemed an ultimate solution in terms of high dynamic response and best disturbance rejection. The special merit of this controller is that it does not require the knowledge of a mathematical model of the plant for the adaptation mechanism, and it can be easily implemented. One effective intelligent search technique, genetic algorithm (GA) [8] is used to optimize the FLC tuning gains speed.

Using the optimization methods based on the mono, multi-objective functions [9-10], or robust methods as Robust $\mathrm{H}$-infinity [11-12]. Our application is based on hybridization between optimization methods and artificial intelligence. The proposed PSS has the ability to adapt its gain in the real time. At first, the system is linearized around an operating point, and then optimizes PSSs settings using the genetic algorithm. Knowing that metaheuristics methods never guarantee optimum found for its development the test will be done to show off the most influencing parameter, by means of this parameter we use fuzzy logic to predict the most influential parameter.

\section{System Modeling}

A power system consists of several elements such as Generators, loads, regulators of (speed, voltage) and control devices, as a whole it present a non-linear system highly complex, this system can be modeled by a set of mechanical and electrical differential equations. The 
single-machine system infinite bus SMIB is the simplest model system generally begins by modeling the system and thereafter, generalizing equations for " $n$ " machines. The system is modeled by nonlinear differential and algebraic equations given in [13]:

$$
\begin{aligned}
\dot{x} & =f(x, y, u) \\
0 & =g(x, y)
\end{aligned}
$$

Where $\mathrm{x}$ is the state vector, $\mathrm{y}$ is the vector of algebraic variables and $\mathrm{g}$ comprises the algebraic values of the stator and the power system equations. As we work on small perturbations, we can linearize the equations of equilibrium point and it lead to the equations:

$$
\begin{aligned}
& \Delta \dot{x}=A \Delta x+B \Delta y+E \Delta u \\
& 0=C \Delta x+D \Delta y
\end{aligned}
$$

A, B, C, D and E are the Jacobins appropriate equations (1) and (2) evaluated at the operating point. For the study of the linearized model, we calculate the eigenvalues or poles of $\lambda_{i}$ system, of the matrix $A$, such as:

$$
\lambda_{i}=\alpha_{i}+j \beta_{i}
$$

The damping factor is given by:

$$
\xi_{i}=\frac{-\alpha_{i}}{\sqrt{\alpha_{i}^{2}+\beta_{i}^{2}}}
$$

This factor allows us to study the stability; if all real part $\alpha_{\mathrm{i}}$ are negative the system is called globally stable, on the contrary if at least one real part of $\alpha_{i}$ is positive the system is said unstable, and on the other hand, if one of eigenvalues by a real part $\alpha_{i}$ which is zero, the answer is not damped. The oscillatory modes are characterized by the oscillating frequency in Hertz given by:

$$
f=\frac{\beta}{2 \pi}
$$

The calculation of the frequency from the eigenvalues of the matrix A closed loop allows us to produce the oscillatory modes of the system. The aim of this paper is to design a PSS which adapts its optimal gain real-time is using fuzzy logic, at first, using a single-machine system for testing the sensitivity parameters of the CPSS (Conventional Power System Stabilizer), to determine the most influential parameter. The second step is to adjust and test the robustness proposed SFPSS. The SFPSS will be subsequently implemented in a multi-machine system for the validation of results. The multimachine system is composed of 3 non-linear synchronous generators and 9 bus (WSCC 3-machine 9-bus), the data of the studied system is cited in [14-15]. Each generation is equipped with AVR (Automatic Voltage Regulator) and a PSS (Power System Stabilizer). The two inputs signals of the devices are injected into the excitation system in Figure 1.

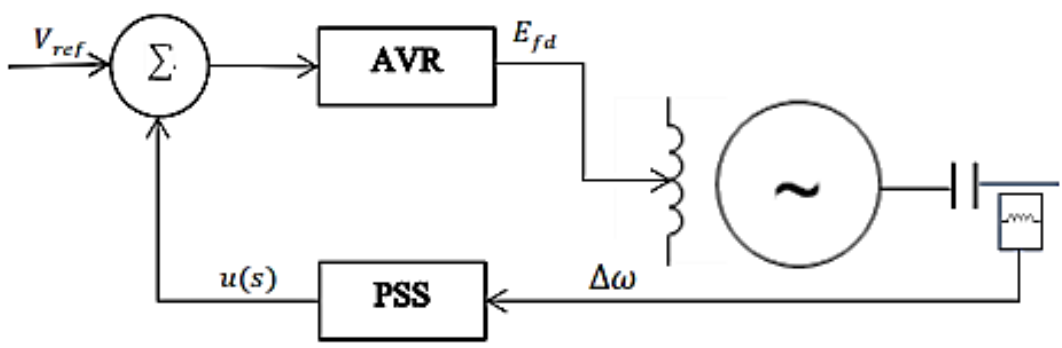

Figure 1. Excitation system "AVR+PSS" 


\section{Power System Stabilizer}

When designing of PSS, it is necessary to respected operating conditions during steady state power system. These aspects suggest that the controls applied to the electrical system must respect some rules. We must follow the first small swing after a major disturbance respecting a certain degree of security, provide a minimum level damping in the equilibrium state after a major disturbance, and reduce the risk of adverse side effects [16]. The block diagram CPSS lead/lag is presented in Figure 1. The main role of the PSS is to introduce a damping torque component to compensate for the one introduced by the AVR. The input signal selected is the variation of the speed difference $\Delta \omega$. Like indicated in Figure 1 , the PSS includes three blocks, the first (1) block is the gain; determining damping quality introduced by the PSS, the second block is a high pass filter (washout) is used to block frequencies below $0.1 \mathrm{~Hz}, T_{\omega}$ value can vary from 5 to 20 seconds, usually $T_{\omega}=10 \mathrm{~s}$. The block (3) is used in the appropriate phase compensation to correct the phase lag between the input of the exciter and the electric torque, finally a voltage limiter has prevented that the PSS disrupts the input of the AVR. Figure 2 shows design of fuzzy logic.

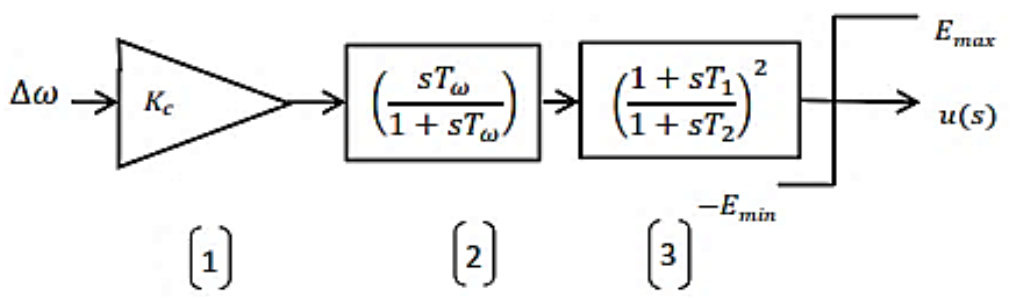

Figure 2. Structure of conventional power system stabilizer

\section{Fuzzy Logic Control}

In particular, fuzzy logic systems (FLS) have been successfully applied to control complex or ill-defined processes whose mathematical models are difficult to obtain. The ability of converting linguistic descriptions into automatic control strategy has made it a practical and promising alternative to the classical control scheme for achieving control of complex nonlinear systems. FLC has two inputs and one output. For convenience, the inputs and output of the FLC are scaled with three different coefficients $k_{e}, k_{\Delta e}$ and $k_{\Delta P}$. Figure 3 shows design of fuzzy logic.

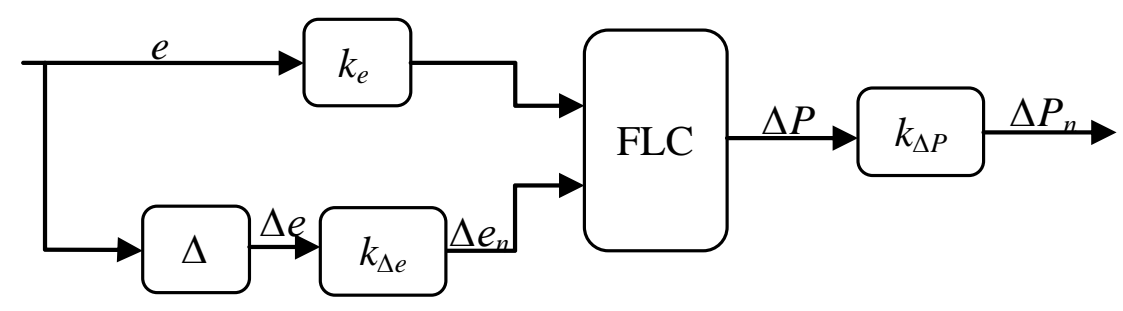

Figure 3. Design of fuzzy logic

\section{Proposed Fuzzy Power System Stabilizer}

In the first place, we must test the sensitivity of each parameter of PSS to see the influence brought by the PSS for maintaining angular stability. The sensitivity is tested on single machine system shown in Figure 4. 


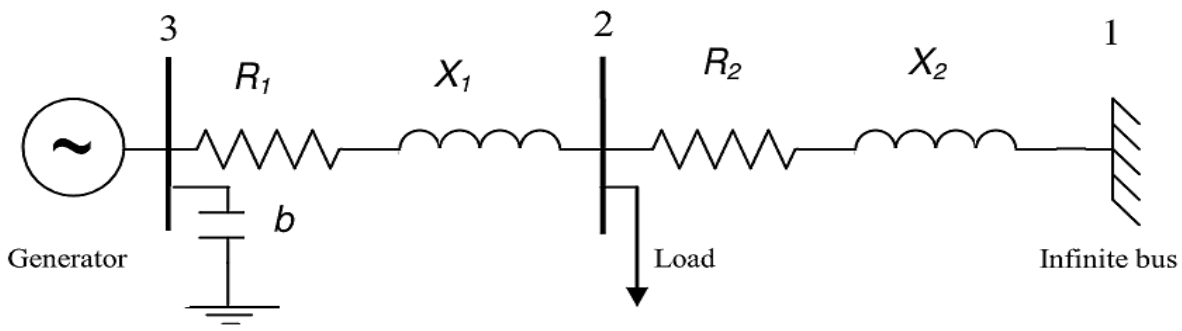

Figure 4. A single machine-infinite-bus power system

The choice of the parameter to be set in this technique depends on the speed sensitivity of these three parameters. For the determination of the sensitivity of the speed of the three key parameters $\mathrm{K}_{\mathrm{c}}, \mathrm{T}_{1}$ and $T_{2}$, in calculating each performance index by the square error (ISE) calculated by the equation:

$$
I S E=\int_{t_{0}}^{t_{f}} \Delta \omega^{2} d t
$$

The speed dynamic error has been calculated in the case of a three-phase short circuit fault of 95 ms that occurs at $t=1 \mathrm{~s}$ applied to the bus number 2 of the SMIB. To determine this sensitivity index, the parameters were globally optimized by the genetic algorithm on the basis of an eigenvalue analysis in a first step. The obtained values are the following:

$$
K_{C}=40.25 ; \mathrm{T}_{1}=0.201 \mathrm{~s} \text { and } \mathrm{T}_{2}=0.078 \mathrm{~s}
$$

Let us now, determine the speed sensitivity to these three key parameters, $\mathrm{K}_{\mathrm{c}}, \mathrm{T}_{1}$ and $T_{2}$ by varying them 0 . This result proves that the Gain has the greatest influence. Table 1 shows speed sensitivity to $\Delta K_{c}, \Delta T_{1}$ and $\Delta T_{2}$ variation.

\begin{tabular}{|c|c|c|c|c|c|c|c|}
\hline$\Delta K_{c}, \mathrm{~T}_{1} \mathrm{~T}_{1}$ and $\Delta T_{2}(\%)$ & -70 & -50 & -10 & 0 & 10 & 50 & 70 \\
\hline $\begin{array}{l}\Delta / S E(\%) \\
=f 1\left(\Delta K_{c}\right)\end{array}$ & -74.38 & -26.10 & -1.72 & 0 & $\begin{array}{c}1.5 \\
0\end{array}$ & 25.17 & 75.65 \\
\hline $\begin{array}{l}\Delta I S E(\%) \\
=f 2\left(\Delta T_{1}\right)\end{array}$ & -35.32 & -16.15 & -0.93 & 0 & $\begin{array}{c}0.6 \\
7\end{array}$ & 15.27 & 49.05 \\
\hline $\begin{array}{l}\Delta / S E(\%) \\
=f 3\left(\Delta T_{2}\right)\end{array}$ & -16.25 & -8.01 & -0.83 & 0 & $\begin{array}{c}0.7 \\
3\end{array}$ & 7.98 & 18.40 \\
\hline
\end{tabular}

Table 1. Speed Sensitivity to $\Delta \boldsymbol{K}_{c}, \Delta \boldsymbol{T}_{1}$ and $\Delta \boldsymbol{T}_{2}$ Variation

Here is the representation of the variation of ISE according to three parameters of PSS. 


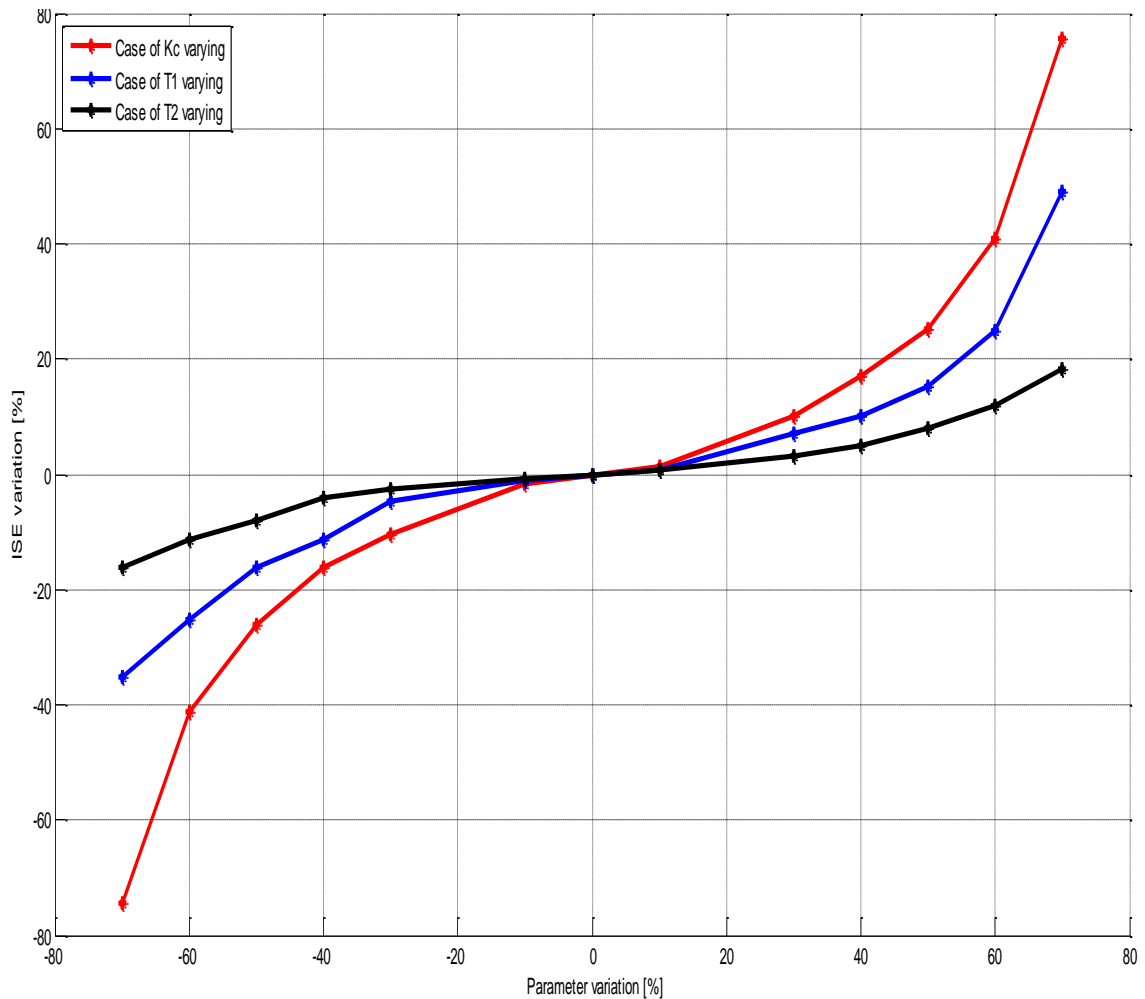

Figure 5. Sensitivity of three parameters according to their variations

\section{Design Methodology and Simulation Studies}

The design steps of the proposed PSS are:

\subsection{Global Optimization of PSS Parameters}

By genetic algorithm (GA), we optimize PSSs parameters for three operating points, light, nominal and Heavy, with an objective function. In this work, the objective function is to damp the maximum electromechanical oscillations, the function is formulated as following: Maximize:

$$
G\left(K_{c p}, T_{1 p} T_{2 p}\right)=\min \left(\min \left(\epsilon_{k}\right)\right) k=1, \ldots n
$$

Subject of:

$$
\begin{aligned}
& 0.01 \leq K_{c i} \leq 50 \\
& 0.01 \leq T_{1 i} \leq 2 s \\
& 0.01 \leq T_{2 i} \leq 2 s \\
& K_{c 1}=K_{c 2}=K_{c 3} \\
& T_{11}=T_{12}=T_{13} \\
& T_{21}=T_{22}=T_{23}
\end{aligned}
$$

where: $\epsilon_{k}$ : Vector of the damping factors of the k operating points.

$n$ : Number of operating point;

$p:$ The index of the PSS.

\subsection{Implementation of Fuzzy Controller}

Using the Toolbox-Matlab by introducing the fuzzy rules, by the fuzzy logic system, using expertise on the gain variation expressed as a rule base. The command is the rotor speed deviation $(d w)$ is selected as inputs and the output signal is the gain variation $\Delta K_{c}$. The 
controller parameters should be determined by trial and error calculation, using the simulation system. Five fuzzy subsets were used in our case. For each of these fuzzy subsets, the triangular membership function (MF) was used. The results through these fuzzy subsets to compute the output is shown in 0 . Table 2 shows rules for fuzzy gain. Table 3 shows fuzzy sets of a variable.

Table 2. Rules for Fuzzy Gain

\begin{tabular}{ccccc}
\hline$d \dot{w} / d w$ & NB & NS & EZ & PS \\
\hline NB & NB & NB & NM & NS \\
NS & NB & NM & NS & EZ \\
EZ & NM & NS & EZ & PS \\
PS & NS & EZ & PS & PM \\
PB & EZ & PS & PM & PB \\
\hline
\end{tabular}

Table 3. Fuzzy Sets of a Variable

\begin{tabular}{cc}
\hline NB & Negative Big \\
\hline NS & Negative Small \\
\hline EZ & Equal Zero \\
\hline PS & Positive Small \\
\hline PM & Positive Medium \\
\hline PB & Positive Big \\
\hline
\end{tabular}

The set of fuzzy rules can generate the control surface that explains the behavior of the variables of the input and output system Figure 6.

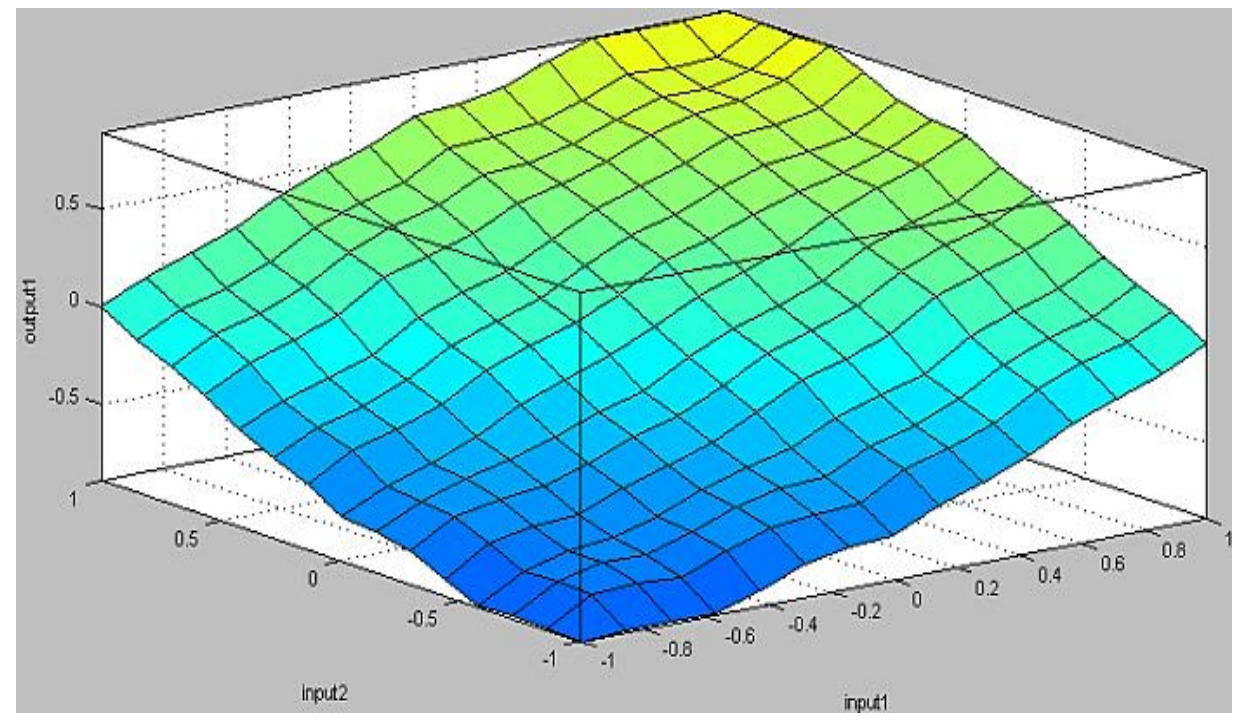

Figure 6. Fuzzy rules control surface

The results given by the genetic algorithm are as follows:

$$
\begin{aligned}
& K_{c(p=1,2 \text { and } 3)}=45.1078 \\
& T_{1(p=1,2 \text { and } 3)}=0.277 \mathrm{~s} \\
& T_{2(p=1,2 \text { and } 3)}=0.005 \mathrm{~s}
\end{aligned}
$$

Using Simulink Matlab, fuzzy adaptive PSS scheme is shown in Figure 7. 


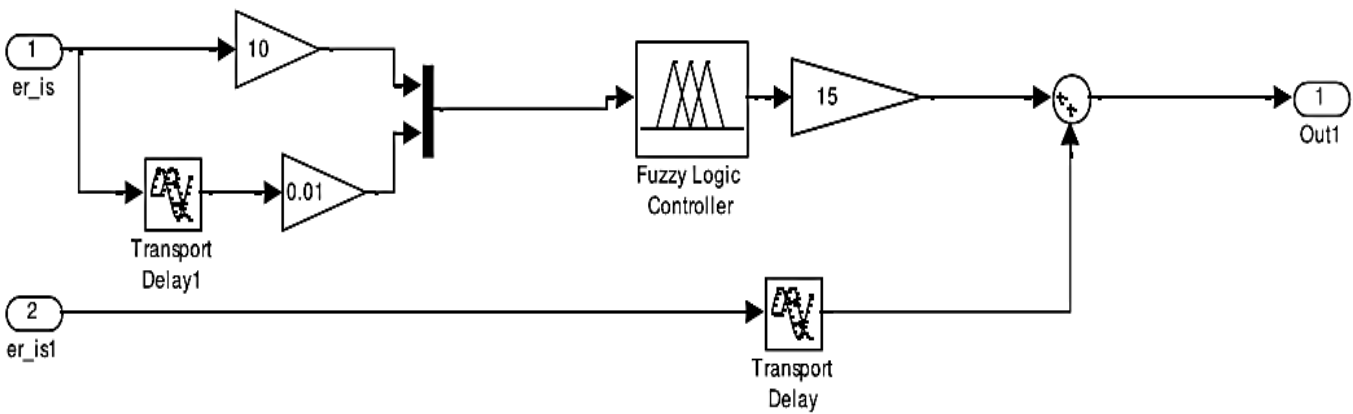

Figure 7. Diagram of fuzzy adaptive gain

After several tests the determined parameters, $k_{e}, k_{\Delta e}$ and $k_{\Delta P}$, we found the following values:

$$
k_{e}=10, k_{\Delta e}=0.01 \text { and } k_{\Delta P}=15 .
$$

For testing the effectiveness of the proposed fuzzy PSS we produce a fault three-phase short circuit at time $\mathrm{t}=1 \mathrm{~s}$ a short circuit duration equal to $50 \mathrm{~ms}$. Simulation results are presented in Figure 8 (a), (b) and (c).

The Table 4 shows a comparison between the results of minimum damping coefficients for each load, these results demonstrate the effectiveness and superiority of the proposed PSS.

Table 4. Minimum/Maximum Damping Coefficients for Each Loading Condition

\begin{tabular}{cccccc}
\hline & & Without & GA PSS & $\begin{array}{c}\text { Fuzzy } \\
\text { PSS }\end{array}$ & \\
\hline Light & Minimum & 0.0026 & 0.0670 & 0.5140 & $\times 10^{-11}$ \\
loading & Maximum & 9.7655 & 29.2659 & 50.1050 & \\
Nominal & Minimum & 0.0013 & 0.0654 & 0.3024 & $\times 10^{-11}$ \\
loading & Maximum & 11.7521 & 30.5726 & 52.1688 & \\
Heavy & Minimum & 0.0041 & 0.0674 & 0.2860 & $\times 10^{-11}$ \\
loading & Maximum & 11.9351 & 30.7773 & 52.5968 & \\
\hline
\end{tabular}

In the following Table 5 we present performance index system optimized by the genetic algorithm and fuzzy system. The results show the great improvement brought by the fuzzy gain system.

Table 5. Amortization Time

\begin{tabular}{lcccc}
\hline & & GA PSS & Fuzzy PSS & Improvement [\%] \\
\hline \multirow{4}{*}{ Generator 1 } & Light & 3.05 & 0.95 & 68,85 \\
& Nominal & 3.00 & 2.88 & 6,67 \\
& Heavy & 2.50 & 1.50 & 40 \\
Generator 2 & Light & 3.10 & 1.05 & 66,13 \\
& Nominal & 2.90 & 1.98 & 31,72 \\
& Heavy & 3.60 & 1.88 & 47,77 \\
Generator 3 & Light & 3.3 & 1.5 & 54,54 \\
& Nominal & 2.86 & 2.25 & 21,32 \\
& Heavy & 2.62 & 2.02 & 22,90 \\
\hline
\end{tabular}

The results of the damping time prove that the control by the fuzzy gain greatly improves the damping time. 

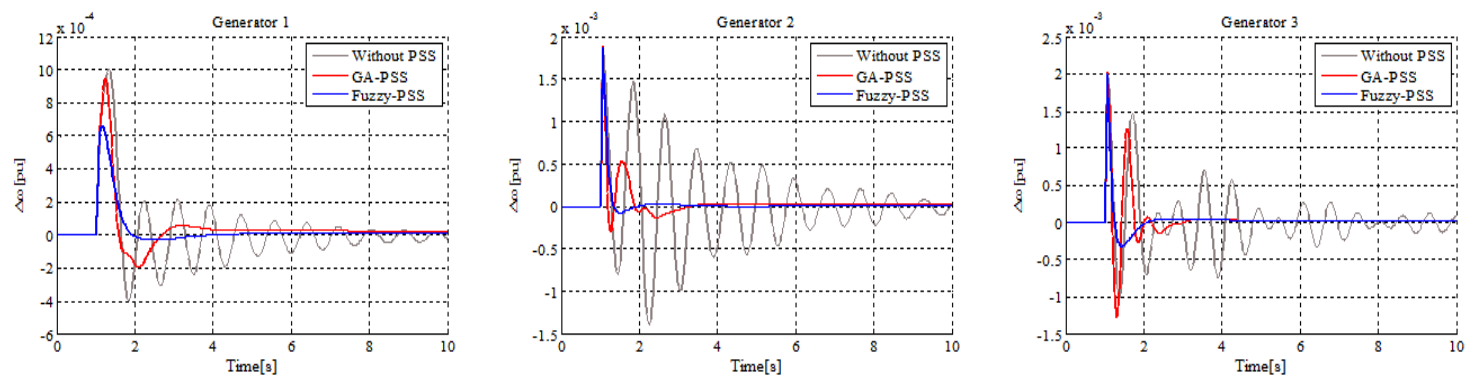

(a) light loading condition
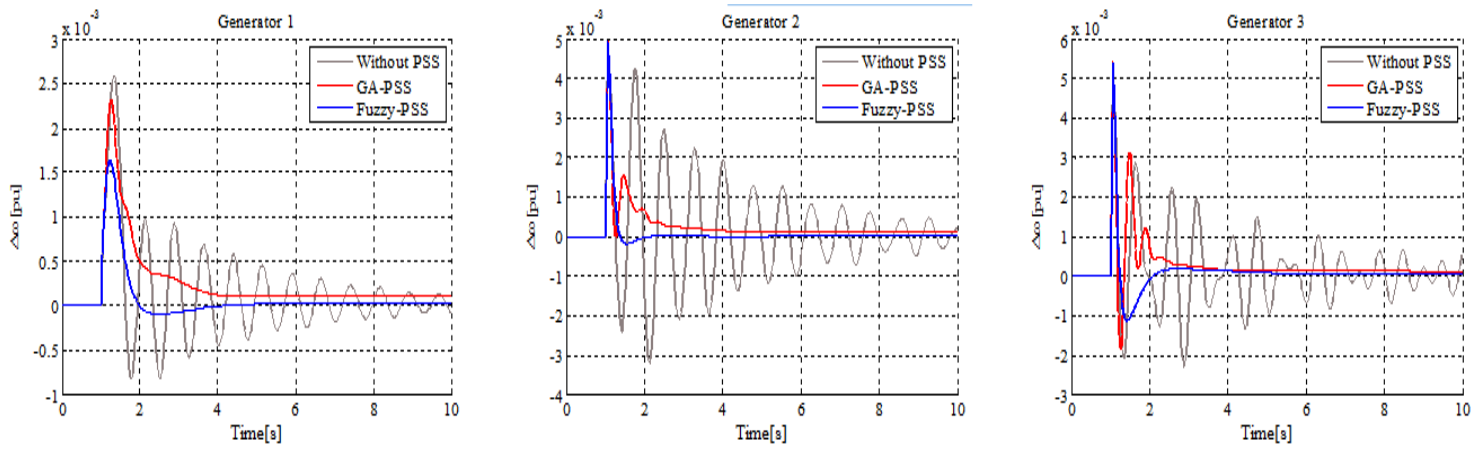

(b) nominal loading condition
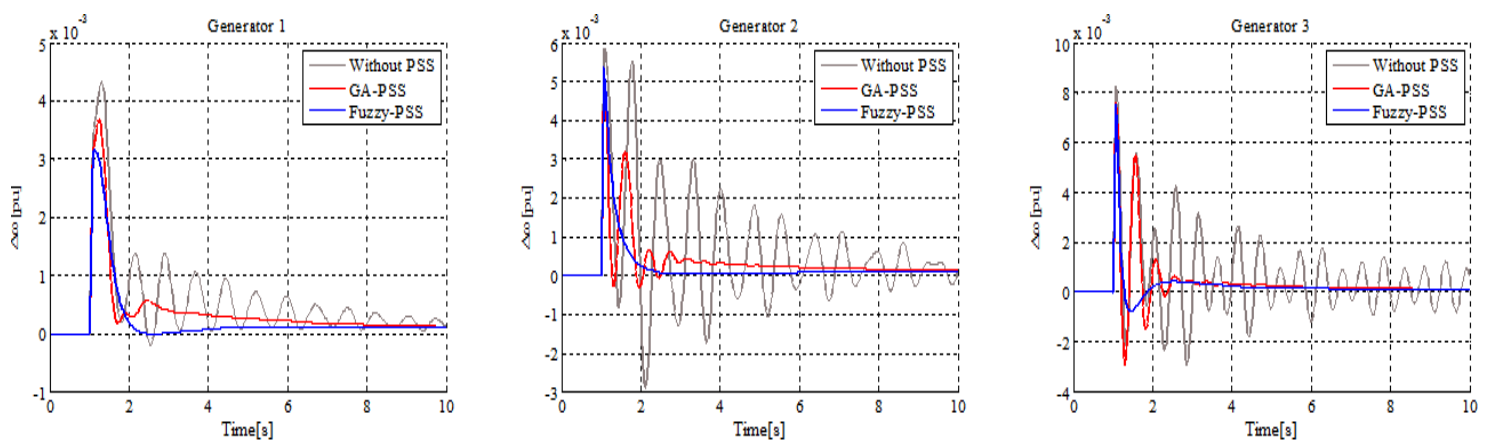

(c) heavy loading condition

Figure 8. Dynamic speed deviations of the three generators of the WSCC power system

\section{Conclusion}

The paper presented an Adaptive Fuzzy Gain of Power System Stabilizer to improve the global stability. For testing the effectiveness of the proposed fuzzy PSS, we produced a fault three-phase short circuit. A comparative study between the conventional GA and the adaptive gain fuzzy of Power System Stabilizer was presented. The overall system (FLC-PSS) performance has been investigated at different dynamic operating conditions. It is concluded that the proposed controller shown superior performances, and robust stability in spite of the presence of a fault three-phase short circuit.

\section{References}

[1] O Roy et MC Maina. Effect of AVR and PSS on Power System Transient Stability with Different Wind Generation Technologies. Journal of Energy Technologies and Policy. 2015; 5(3): 66-71.

[2] B Singh Surjan et R Garg. Power System Stabilizer Controller Design for SMIB Stability Study. International Journal of Engineering and Advanced Technology. 2012; 2(1): 209-2014. 
[3] C Phil-Hun, S Myong-Chul, K Hak-Man and C Jae-Sang.A Study of Power Network Stabilization Using an Artificial Neural Network. Knowledge-Based Intelligent Information and Engineering Systems, Springer Berlin Heidelberg, 2005: 479-484. DOI:10.1007/11552413_69

[4] W Liu, GK Venayagamoorthy et DC Wunsch. Adaptive Neural Network Based Power System Stabilizer Design. Neural Networks. Elsevier. 2003; 16(5-6): 891-898. DOI:10.1109/IJCNN.2003.1224043

[5] AM Sharaf et TT Lie. A hybrid neuro-fuzzy power system stabilizer. Neural Networks, 1994. IEEE World Congress on Computational Intelligence. 1994 IEEE International Conference, Orlando, FL, 1994. DOI: $10.1109 /$ ICNN.1994.374422

[6] HE Talaat, A Abdennour et AA Al-Sulaiman. Design and experimental investigation of a decentralized GA-optimized neuro-fuzzy power system stabilizer. Electrical Power and Energy Systems. 2010; 32 : 751-759. DOI 10.1016/j.ijepes.2010.01.011

[7] UR Babu, VV Kumar Reddy et ST Kalyani. Design of Power System Stabilizer with Neuro-Fuzzy UPFC Controller. International Journal of Electrical, Computer, Energetic, Electronic and Communication Engineering. 2014; 8(12): 1945-1948. scholar.waset.org/1999.0/10002786

[8] KM Passino, S Yurkovich. Fuzzy Control. Addison Wesley Publishing Company, 1997.

[9] M Farahani. A Multi-Objective Power System Stabilizer. IEEE Transactions on Power Systems. 2013; 28(3): 2700-2707.

[10] AJ Urdaneta, NJ Bacalao, B Feijoo, L Flores et R Diaz. Tuning of power system stabilizers using optimization techniques. IEEE Transactions on Power Systems. 1991; 6(1): 127-134. DOI: $10.1109 / 59.131055$

[11] Y Peng, QM Zhu et H. Nouri. Robust H2 power system stabilizer design using LMI techniques. Modelling, Identification and Control (ICMIC), Proceedings of 2011 International Conference, Shanghai. 2011. DOI: 10.1109/ICMIC.2011.5973739

[12] R Kutzner. A robust $\mathrm{H}_{\infty}$ PSS with enlarged damping range. Power Engineering Society Summer Meeting, 1999. IEEE, Edmonton, Alta. 1999; 1: 53-57. DOI: 10.1109/PESS.1999.784324

[13] PW SAUER et MA PAI, Power System Dynamics and Stability, New Jersey: Upper Saddle River, 1998.

[14] MA Abido. Optimal Design of Power-System Stabilizers Using Particle Swarm Optimization. IEEE Transactions on Energy Conversion. 2002; 17(3): 406 - 413. DOI: 10.1109/TEC.2002.801992

[15] K Gurpreet et B Navneet Singh. Transient Stability Analysis of IEEE 9 Bus System Using Power World Simulator. International Journal of Advanced Research in Electrical, Electronics and Instrumentation Engineering. 2016; 5(4): 2632-2639. DOI:10.15662/IJAREEIE.2016.0504083

[16] RG Farmer. Power System Dynamics and Stability. The Electric Power Engineering Handbook, Ed. L.L. Grigsby. 2001. 